\title{
Applied differential equations and related computational mathematics in chemistry
}

\author{
J. Vigo-Aguiar
}

Published online: 1 March 2014

(C) Springer International Publishing Switzerland 2014

We are pleased to offer readers of the Journal of Mathematical Chemistry this Special Issue consisting of papers on the topics discussed at the 13th International Conference of Computational and Mathematical Methods in Science and Engineering, CMMSE_-2013, held at Almeria, Spain, June 24-28, 2013. This special issue includes not only papers actually presented at the Conference, but also some additional, specially selected papers, which are thematically related to the subjects discussed at the conference, and provide additional overview and a fuller coverage of the field typically covered by the CMMSE meetings.

Since its foundation in Milwaukee in 2000, the CMMSE organization has provided a stimulating annual forum for researchers involved in some of the interdisciplinary areas of applied mathematics and natural sciences, especially, chemistry. Most of these researchers and contributors have found in CMMSE a fruitful arena in which to disseminate their results and advances to the research community.

The Applied and Computational Mathematics topics of CMMSE are methods and techniques primarily in the following fields: differential equations, approximation theory, asymptotic methods, variational methods, and numerical analysis. These areas of applied mathematics are intimately related to the development of both Mathematical Chemistry and Computational Chemistry.

In particular, the mathematical chemistry components of several of the papers selected for this issue correspond to the development of numerical methods for several variants of the Schrödinger equation. This family of differential equations appears often in scientific areas such as quantum chemistry, molecular physics, but also in nuclear physics. During the past three decades, a significant body of research has been carried out on the numerical solution of these Schrödinger equations, in addi-

\footnotetext{
J. Vigo-Aguiar $(\bowtie)$

Department of Applied Mathematics, University of Salamanca, Salamanca, Spain e-mail: jvigo@usal.es
} 
tion to some more general ordinary and partial differential equations with oscillatory character.. Approximate solutions to the Schrödinger equations are arguably the most important for mathematical chemistry. Here, the crucial issues are high accuracy of the approximation and the size of the described systems; these issues are still unresolved and turn out to be particularly challenging.

In this special issue, some mathematical techniques for numerical solutions are also considered; however, there are also new developments in the area of analytical solutions, including, for example, the $\mathrm{N}$-dimensional Schrödinger equation with the Morse potential, with approaches based on the Laplace transformation method. Other choices of solutions, such as the series solution methods have also been explored in this issue. A less common approach, also useful for the study of the global behavior of certain chemical systems, involves the use of dynamical system techniques and is applied in a study of the decomposition of pseudo-radioactive products that follow a dynamics determined by a trigonometric factor.

A related approach can be used in the search for fundamental parameters involved in $\mathrm{X}$-ray fluorescence analysis. In order to obtain the concentrations of chemical elements in a sample it is necessary to calculate some integrals of the spectral distribution of the fluorescence exciting X-ray beam, where the determination of a weighting function is essential.

New techniques of optimization have also been introduced, for example, a method designed for a constrained optimal control problem. This method calculates the optimal enzyme concentrations in a chemical process based on the minimization of the transition time.

In summary, this collection of articles is expected to be useful and informative concerning some of the new perspectives and trends in the interactions between Chemistry and Mathematics, and I hope that readers will enjoy learning about these results, which I hope will also serve as inspiration for further advances.

Finally, I want to thank all the authors and referees for their inspired and valuable contributions to this special volume. 